\title{
The Afterglows of Gamma-Ray Bursts
}

\author{
S. R. Kulkarni*, E. Berger*, J. S. Bloom*, F. Chaffee", \\ A. Diercks*, S. G. Djorgovski*, D. A. Frail ${ }^{\dagger}$, T. J. Galama*, \\ R. W. Goodrich , F. A. Harrison*, R. Sari*, and S. A. Yost* \\ *California Institute of Technology, Pasadena, CA 91125, USA \\ ${ }^{\dagger}$ National Radio Astronomy Observatory, Socorro, NM 87801, USA \\ ${ }^{\top}$ W. M. Keck Observatory, Kamuela, HI 96743, USA
}

\begin{abstract}
Gamma-ray burst astronomy has undergone a revolution in the last three years, spurred by the discovery of fading long-wavelength counterparts. We now know that at least the long-duration GRBs lie at cosmological distances with estimated electromagnetic energy release of $10^{51}-10^{53} \mathrm{erg}$, making these the brightest explosions in the Universe. In this article we review the current observational state, beginning with the statistics of X-ray, optical, and radio afterglow detections. We then discuss the insights these observations have given to the progenitor population, the energetics of the GRB events, and the physics of the afterglow emission. We focus particular attention on the evidence linking GRBs to the explosion of massive stars. Throughout, we identify remaining puzzles and uncertainties, and emphasize promising observational tools for addressing them. The imminent launch of HETE- 2 and the increasingly sophisticated and coordinated ground-based and space-based observations have primed this field for fantastic growth.
\end{abstract}

\section{INTRODUCTION}

GRBs have mystified and fascinated astronomers since their discovery. Their brilliance and their short time variability clearly suggest a compact object (black hole or neutron star) origin. Three decades of high-energy observations, culminating in the definitive measurements of CGRO/BATSE, determined the spatial distribution to be isotropic yet inhomogeneous, suggestive of an extragalactic population (see [14] for a review of the situation prior to the launch of the BeppoSAX mission). Further progress had to await the availability of GRB positions adequate for identification of counterparts at other wavelengths.

In the cosmological scenario, GRBs would have energy releases comparable to those of supernovae (SNe). Based on this analogy, Paczyński \& Rhoads [65] and Katz [44] predicted that the gamma-ray burst would be followed by long-lived but fading emission. These papers motivated systematic searches for radio afterglow,

CP526, Gamma-Ray Bursts: $5^{\text {th }}$ Huntsville Symposium, edited by R. M. Kippen, et al.

(C) 2000 American Institute of Physics 1-56396-947-5/00/\$17.00 
including our effort at the VLA [15]. The broad-band nature of this "afterglow" and its detectability was underscored in later work $[59,78]$.

Ultimately, the detection of the predicted afterglow had to await localizations provided by the Italian-Dutch satellite, BeppoSAX [6]. The BeppoSAX Wide Field Camera (WFC) observes about $3 \%$ of the sky, triggering on the low-energy (2-30 $\mathrm{keV}$ ) portion of the GRB spectrum, localizing events to $\sim 5-10$ arcminutes. X-ray afterglow was first discovered by BeppoSAX in GRB 970228, after the satellite was re-oriented (within about 8 hours) to study the error circle of a WFC detection with the 2-10 keV X-ray concentrators. The detection of fading X-ray emission, combined with the high sensitivity and the ability of the concentrators to refine the position to the arcminute level, led to the subsequent discovery of long-lived emission at lower frequencies $[10,77,16]$.

Optical spectroscopy of the afterglow of GRB 970508 led to the definitive demonstration of the extragalactic nature of this GRB [60]. The precise positions provided by radio and/or optical afterglow observations have allowed for the identification of host galaxies, found in almost every case. Not only has this provided further redshift determinations, but it has been useful in tying GRBs to star formation through measurements of the host star formation rate (e.g., $[46,11])$. HST, with its exquisite resolution, has been critical in localizing GRBs within their host galaxies and thereby shed light on their progenitors (e.g., $[29,41,4])$ ). Observations of the radio afterglow have directly established the relativistic nature of the GRB explosions [16] and provided evidence linking GRBs to dusty star-forming regions. Radio observations are excellent probes of the circumburst medium and the current evidence suggests that the progenitors are massive stars with copious stellar winds. The latest twist is an apparent connection of GRBs with SNe [5]. Separately, an important development is the possible association of a GRB with a nearby (40 Mpc) peculiar SN $[30,47]$.

In this paper we review the primary advances resulting from afterglow studies. $\S I I$ discusses the statistics of detections to-date, including possible causes for the lack of radio and optical afterglows from some GRBs. In §III we review constraints on the nature of the progenitor population(s), in particular evidence linking some classes of GRBs to SNe. §IV describes the status of current understanding of the physics of the afterglow emission. Here we compare observations to predictions of the basic spherically-symmetric model, and describe complications arising from deviations from spherical symmetry and non-uniform distribution of the circumburst medium. We conclude with speculations of the near- and long-term advances in this field $(\S \mathrm{V})$.

We point out that this review has two biases. First, given the concentration of previous review articles on optical and X-ray observations, we emphasize the unique contributions of radio afterglow measurements. Second, this article is intended to also provide a summary of the efforts of the Caltech-NRAO-CARA GRB collaboration, and therefore details our work in particular. This review is in response to review talks given at the 1999 Maryland October meeting (SRK) and the 5th Huntsville GRB meeting (DAF and SRK). 

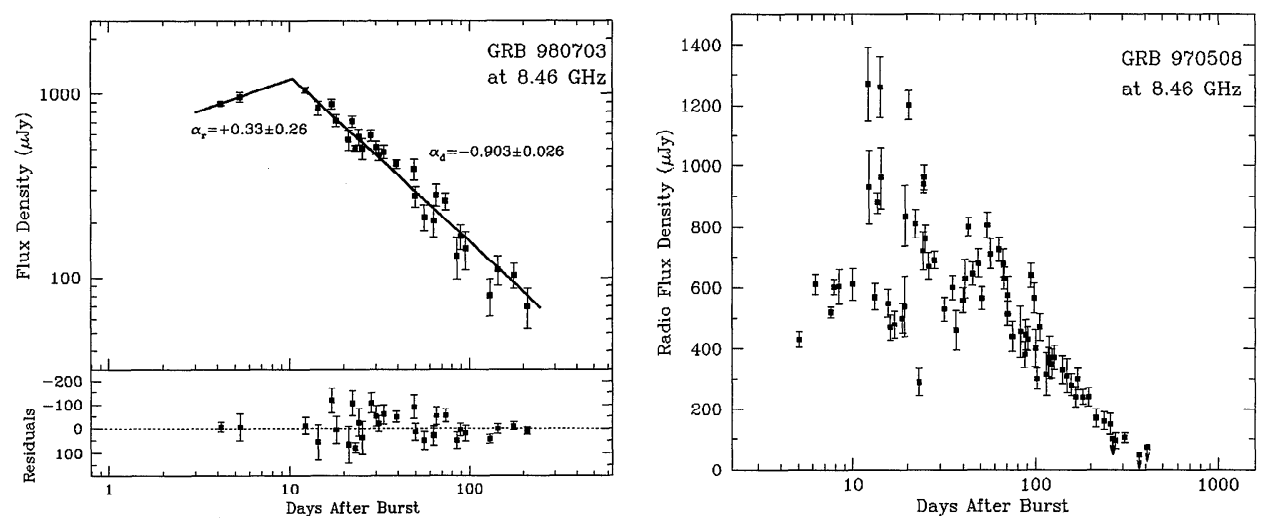

FIGURE 1. Left: The radio light curve of GRB 980703. This is a typical afterglow, a rise to a peak followed by a power-law decay. The longer lifetime of the radio afterglow allows us to see both the rise and the fall of the afterglow emission. In contrast, at optical and X-ray emission, most of the times we see only the decaying portion of the light curve. Right: The radio light curve of GRB 970508 [21]. The wild fluctuations of the light curve in the first three weeks are chromatic. At later times, the fluctuations become broad-band and subdued. These fluctuations are a result of multi-path propagation of the radio waves in the Galactic interstellar medium. As the source expands (at superluminal speeds) the scintillation changes from diffractive to refractive scintillation. This is analogous to why stars twinkle but planets do not.

\section{STATISTICS OF AFTERGLOW DETECTIONS}

Afterglow emission was first detected from GRB 970228, both at X-ray [10] and optical frequencies [77], but not at radio wavelengths [17]. The first radio afterglow detection came following the localization of GRB 970508 [16]. Figure 1 shows two examples of radio lightcurves. The radio afterglow of GRB970508 is famous for several reasons: it was the first radio detection, it gave the first direct demonstration of relativistic expansion, and it remains the longest-lived afterglow [21].

Afterglow emission is now routinely detected across the electromagnetic spectrum. BeppoSAX has been joined in studying the X-ray afterglows by the All Sky Monitor (ASM) aboard the X-ray Timing Explorer (XTE), the Japanese ASCA mission, and recently the Chandra X-ray observatory (CXO). A veritable armada of optical facilities (ranging from 1-m class telescopes to the 10-m Keck telescopes) routinely discover and study optical afterglows. HST has been primarily used to make exquisite images of thc host galaxies (see above) but in the near future we expect other uses such as UV spectroscopy and identification of underlying SNe. The VLA has led the detection in radio. However, other centimeter-wavelength facilities (the Australia Telescope National Facility, Westerbork Synthesis Radio Telescope, the Ryle Telescope) and millimeter wavelengths (James Clerk Maxwell Telescope, the Owens Valley Millimeter Array, IRAM and the Plateau de Bure 
Interferometer) are now regularly contributing to afterglow studies.

Figure 2 summarizes the statistics of afterglow detections. In almost all cases, $\mathrm{X}$-ray emission has been detected, establishing the critical importance of prompt $\mathrm{X}$-ray observations. Optical afterglow appears to be detected in about $2 / 3$ of all well-localized events if sufficiently deep optical images are taken rapidly (i.e., within a day or so of the burst). Radio afterglows are detected in $40 \%$ of the cases - far more often than usually assumed. We refer the reader to the Frail et al. [22] for a comprehensive summary of the X-ray/optical/radio afterglow detection statistics. The non-detections are, as discussed below, as interesting as the detections.

\section{Venn Diagram for GRB Afterglows}

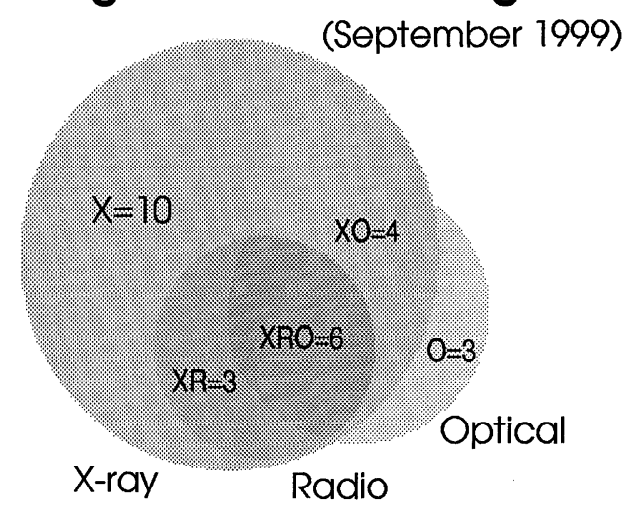

FIGURE 2. A Venn diagram showing the detection statistics for 26 well-localized GRBs in the Northern and Southern hemispheres. Of the 23 GRBs for which X-ray afterglows have been detected to date, 10 have optical afterglows $(X O+X O R)$ and 9 have radio afterglows (XR + $X O R)$. In total there are 13 optical and/or radio afterglows with corresponding $X$-ray afterglows.

Radio Non-detection. The failure to find radio afterglow is most likely due to lack of sensitivity. The brightest radio afterglow to date is that from GRB 991208 (Frail GCN ${ }^{1}$ 451) with a peak flux of $2 \mathrm{mJy}$, a $60-\sigma$ detection (at centimeter wavelengths), whereas the weakest afterglow is typically around $5 \sigma$. In contrast, at optical and X-ray wavelengths, afterglow emission is routinely detected at hundreds of sigma. If the VLA were to be upgraded by a factor of 10 in sensitivity, then we predict that radio afterglow emission would, like X-ray emission, be detected from most GRBs.

Optical Non-detection. Non-detection at optical wavelengths is more interesting, as it may result in some cases from extinction along the line of sight or within the source. Bad weather as well as rapid fading of the afterglow has certainly hindered some optical searches, which, due to notification delays, typically begin some

1) GCN refers to the GRB Coordinates Network Circular Services. This network is maintained by S. Barthelmy at the Goddard Space Flight Center; see http://gcn.gsfc.nasa.gov/gcn/ 
hours after the event. Furthermore, low Galactic latitude events may be obscured, or hidden in crowded foregrounds. However, in some cases deep searches have been performed with no success. Here, non-detection likely results from extinction by dust in the burst host galaxy and/or absorption by the intergalactic medium. GRB 970828 [38] is one example, as is the more dramatic case of GRB 980329. This burst was one of the brightest events in the WFC [42]. Searches for optical afterglow emission failed to identify any counterpart. VLA observations identified an unusual radio variable in the field [76]. Soon thereafter, a red afterglow and a bright IR afterglow were identified (Klose GCN 43, Larkin et al. GCN 44). Taylor et al. [76] suggest that the GRB arose in a region with high extinction. Further optical and IR work on this interesting afterglow can be found in $[34,64,69]$.

Optically dim "red" but bright IR afterglows can also result from the GRB being located at high redshift. Intergalactic $\mathrm{HI}$ absorption will result in a wavelength cutoff below the Lyman limit, $<912(1+z) \AA$, where $z$ is the redshift of the source. This effect was originally invoked to explain the faint R-band but bright IR emission from GRB 980329 [27]. We now know, based on recent Keck observations, that the GRB host is blue, incompatible with a high- $z$ origin. Rather, it is more tenable that the host is a typical star-forming galaxy with dusty star-forming regions, and that the GRB occurred in one such region [76]. We are presently carrying out IR spectroscopy of this host to determine the redshift and the star formation rate (SFR). While searching for " $\mathrm{R}$ dropouts" may in the future provide an effective method for finding high-redshift events, it is clear that cross-calibrated multi-band photometry of higher quality than currently exists will be required to make this useful.

X-ray Non-detection. The spectra of most GRB events clearly extend into the $\mathrm{X}$-ray band, as established by GINGA observations [75]. How the X-ray emission observed during the burst connects to the X-ray afterglow is uncertain, due to sensitivity limitations of wide-field monitors. X-ray afterglow emission appears to be ubiquitous. Observations of the X-ray afterglow are important for two reasons: (i) the observations of the X-ray afterglow by sensitive imaging instruments (e.g., the concentrators aboard BeppoSAX) result in sufficiently precise (arcminute) localization and (ii) a significant (perhaps even a dominant) fraction of the explosion energy appears to be radiated in this band. Of all the SAX bursts, GRB 970111 is peculiar for the absence of X-ray afterglow (admittedly the data were obtained about 17 hours after the burst) [25]. In view of the critical role played by X-ray afterglow in localization of GRBs we regard this non-detection to be worthy of further investigation.

\section{THE NATURE OF THE PROGENITORS}

In almost all cases, a host galaxy has been identified at the location of the fading afterglow. GRB redshifts can be obtained either via absorption spectroscopy (when the transient is bright) or by emission spectroscopy of the host galaxy. In Figure 3 
TABLE 1. Basic Properties of Selected GRBs

\begin{tabular}{lcccccl}
\hline GRB & $\begin{array}{c}\alpha(\mathrm{J} 2000) \\
(\mathrm{h} \mathrm{m} \mathrm{s})\end{array}$ & $\begin{array}{c}\delta(\mathrm{J} 2000) \\
\left(\mathrm{O}^{\prime \prime} \text { ' }\right)\end{array}$ & $\begin{array}{c}\mathrm{R}_{\text {host }} \\
(\mathrm{mag})\end{array}$ & $\begin{array}{c}\mathrm{S} \times 10^{-6} \\
\left(\mathrm{erg} \mathrm{cm} \mathrm{cm}^{-2}\right)\end{array}$ & $z$ & References $^{\mathrm{a}}$ \\
\hline 970228 & 050147 & +1146.9 & $25.2^{\mathrm{b}}$ & 1.7 & 0.695 & Djorgovski et al. GCN 289 \\
970508 & 065349 & +7916.3 & 25.7 & 3.1 & 0.835 & {$[60,2]$} \\
970828 & 180832 & +591852 & $\mathrm{TBD}$ & 74 & 0.957 & {$[12]$} \\
971214 & 115626 & +6512.0 & 25.6 & 11 & 3.418 & {$[46]$} \\
980326 & 083634 & -1851.4 & 227.3 & 1 & $\ldots$ & \\
980329 & 070238 & +3850.7 & 25.4 & 50 & $\ldots$ & \\
980519 & 212221 & +7715.7 & 26.2 & 25 & $\ldots$ & \\
980613 & 101758 & +7127.4 & 24.5 & 1.7 & 1.096 & Djorgovski et al. GCN 189 \\
980703 & 235907 & +0835.1 & 22.6 & 37 & 0.966 & {$[11]$} \\
981226 & 232937 & -235554 & $\gtrsim 22$ & N.A. & $\ldots$ & \\
990123 & 152531 & +444600 & 24.4 & 265 & 1.600 & {$[48]$} \\
990510 & 133807 & -802949 & $Z 28$ & 23 & 1.619 & Vreeswijk et al. GCN 324 \\
990712 & 223153 & -732429 & 21.78 & N.A. & 0.430 & Galama et al. GCN 388 \\
991208 & 163354 & +462721 & $\gtrsim 25$ & 100 & 0.706 & Dodonov et al. GCN 475 \\
991216 & 050931 & +111707 & 24.5 & 256 & 1.020 & Vreeswijk et al. GCN 496 \\
\hline
\end{tabular}

${ }^{a}$ References to redshift determination.

${ }^{\mathrm{b}} V$-band magnitude from HST. All others are $\mathbf{R}$ magnitude in the Johnson system.

and Table 1 we summarize the measured redshifts and host galaxy magnitudes. While the distance scale debate is settled (at least for the class of long-duration GRBs, see below) we remain relatively ignorant of the nature of the central engine. Currently popular GRB models fall into two categories: (i) the coalescence of compact objects (neutron stars, black holes and white dwarfs $[13,54,61,63]$ ) and (ii) the collapse of the central iron core of a massive star to a spinning black hole, a "collapsar" $[85,57]$. We now summarize the light shed on the progenitor problem by afterglow studies.

The Location of GRBs Within Hosts. A fundamental insight into the nature of $\mathrm{SNe}$ came from their location with respect to other objects within the host galaxy (specifically HII regions and spiral arms) and the morphology of the host galaxy itself (elliptical versus spiral). In a similar manner, we are now making progress in understanding GRB progenitors by measuring offsets with respect to other objects in the host galaxies. The rather good coincidence of GRBs with host galaxies already suggests that they are unlikely to be a halo population (as would be expected in the coalescence scenario [3]). On the other hand, with the possible exception of GRB 970508 [66], they are clearly not associated with galactic nuclei (i.e., massive central black holes). Typical offsets of GRBs from the centroid of their host galaxies are comparable to the half-light radii of field galaxies at comparable magnitudes, suggesting that GRBs originate from stellar populations.

Host Galaxies. Demonstrating a direct link between GRBs and (massive) star formation is more difficult. On the whole, the population of identified hosis seems typical in comparison to field galaxies in the same redshift and magnitude range. The hosts have average luminosities for field galaxies, modulo corrections due to 


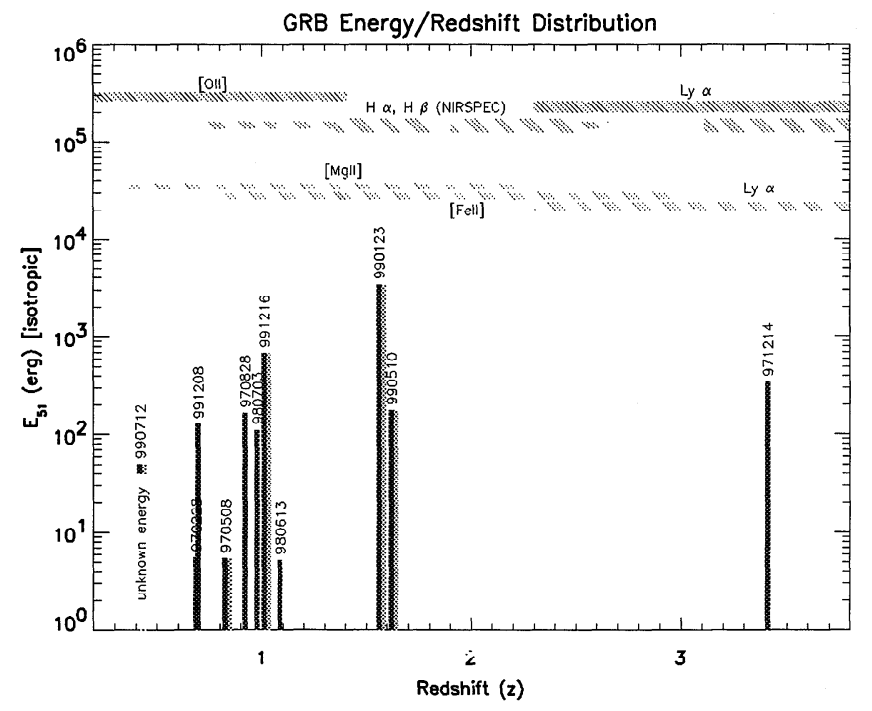

FIGURE 3. The isotropic gamma-ray energy distribution of GRBs with confirmed redshifts. Bursts indicated in black are those with spectroscopically confirmed emission lines from the host galaxies; bursts indicated by a shaded column (e.g., GRB 990123) are those with absorption line redshifts. The relevant key absorption or emission features are noted at the top of the figure.

evolution. Their emission line fluxes and equivalent widths are also statistically indistinguishable from the normal field galaxy population. The observed star formation rates, derived from recombination line fluxes (mostly the [O II] $3727 \AA$ line) and from the UV continuum flux range from less than $1 M_{\odot} \mathrm{yr}^{-1}$ to several tens of $M_{\odot} \mathrm{yr}^{-1}$ - typical of normal galaxies at comparable redshifts (extinction corrections can increase these numbers by a factor of a few, but similar corrections apply to the comparison field galaxy population as well). It will probably be necessary to have a sample of several tens of GRB hosts before a correlation of GRBs with the (massive) star formation rate can be tested statistically. However, below we point to several specific examples that are suggestive of a link between GRBs and star-forming regions.

Association with Starforming Regions. There is evidence showing that GRBs arise from dusty regions within their host galaxies. In this respect, radio observations provide a unique tool for detecting events in regions of high ambient density (as was the case for GRB 980329). An even more extreme example is GRB 970828, where the host was identified based solely on the VLA discovery of a radio flare [12]. Interestingly enough, this is the dustiest galaxy in the sample of GRB hosts to-date.

Second, some GRBs appear to be located within identifiable star-forming regions. An example is GRB 990123 [4,28,41]. VLA observations of GRB 980703 [19] are perhaps more convincing. The radio observations can be sensibly interpreted by 
appealing to free-free absorption from a foreground HII region (which would dwarf the Orion complex). If this interpretation is correct then this would be strong evidence for a GRB being located within a starburst region.

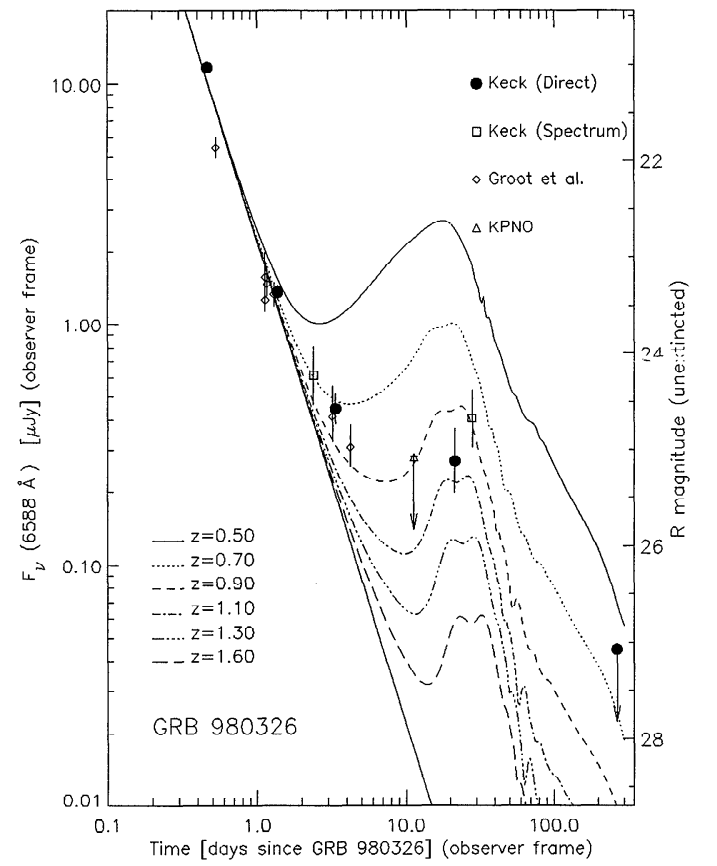

FIGURE 4. R-band light curve of GRB 980326 and the sum of an initial power-law decay plus Ic supernova light curve for redshifts ranging from $z=0.50$ to $z=1.60$ (reprinted by permission from Nature [5] copyright (19g9) Macmillan Magazines Ltd.).

The GRB-SN link. If GRBs arise from the collapse of a massive star, it is an unavoidable consequence that emission from the underlying supernova should be superimposed on the afterglow. Bloom et al. [5] may have made the first detection of a possible SN component in the GRB 980326 lightcurve (Fig. 4). These authors noted that $\mathrm{SNe}$, in contrast to afterglows, have distinctive temporal and spectral signatures: rising to a maximum at $\sim 20(1+z)$ days, with little emission blueward of about $4000 \AA$ in the restframe (and certainly blueward of $3000 \AA$ ) owing to a multitude of resonance absorption lines. This discovery has led to other possible SN detections, most notably GRB $970228[31,68]$.

The suggestion of a GRB-SN connection is an intriguing one but it has yet to be placed on a firm footing. Important questions are: (i) are all long-duration GRBs accompanied by SNe? (ii) if so, are these SNe of type Ib/c? Groundbased observations are possible in those cases where the afterglow decays rapidly (e.g., GRB 980326) or if high quality optical and IR observations exist (e.g., GRB 970228).

We need more examples to test the GRB-SN link. Future progress will depend on a combination of ground and HST observations. For relatively nearby GRBs, especially those with a rapidly decaying optical afterglow, it would be attractive 
and feasible to obtain the spectrum of the SN around the time when the flux from the SN peaks. A moderate quality spectrum with SN-like features would have the singular advantage of definitively confirming the SN interpretation (as opposed to alternatives involving re-radiation by dust [80]). However, for most GRBs, we expect HST observations to play a critical role. HST's widely recognized strengths in accurate photometry of sources embedded in galaxies [32] and photometric stability make the detection of a faint SN against the optical afterglow and the host galaxy possible.

Diversity of the Progenitor Population. As was the case with $\mathrm{SNe}$, it is likely naive to think of a single progenitor population. Below, we discuss the two additional classes which show some promise: the mysterious short duration GRBs and a possible class of low luminosity GRBs associated with SNe.

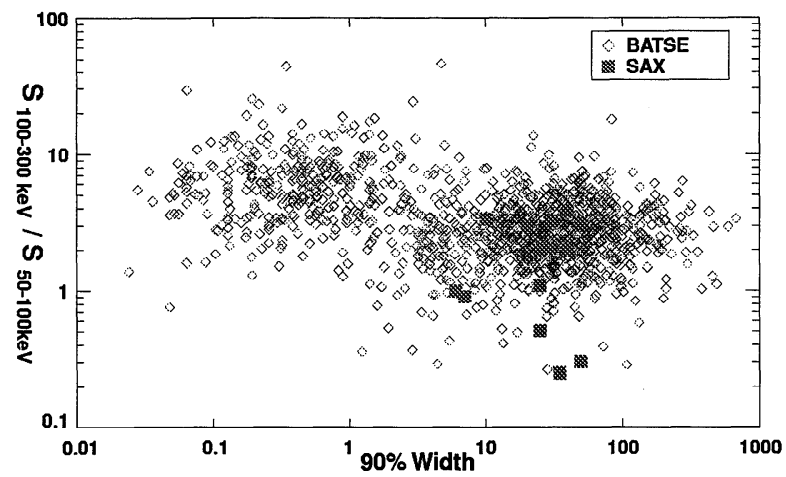

FIGURE 5. Distribution of duration ( $T_{90}$ ) vs. spectral hardness for BATSE bursts (diamonds) from the $4 B$ catalog. There is a clear suggestion of two groups of GRBs: short/hard and long/soft events. Events localized by BeppoSAX (solid squares) appear to belong to the long-duration class.

Short Events. It has been known for some time that the distribution of the duration of GRBs appears to be bimodal [14]; see Figure 5. Furthermore, these two groups may have different spatial distributions [45], with the short bursts being detected out to smaller limiting redshifts. However, we know very little about this class of GRBs since, as noted earlier, all bursts localized by BeppoSAX and RXTE thus far are of long duration (Figure 5). Fortunately, improvements in BeppoSAX and the imminent launch of HETE-2 provide for the first time the opportunity to follow-up short GRBs.

The short duration bursts are difficult to accommodate in the collapsar model, given the long collapse time of the core. However, they find a natural explanation in the coalescence models. How would these bursts manifest themselves? Li \& Paczyǹski [56] speculate that if the short-duration bursts result from NS-NS mergers then they may leave a bright, but short-lived ( $\lesssim 1$ day) optical transient. Radio observations provide a complementary tool for determining the nature of the short duration bursts. The low ambient density would result in weak afterglows (since 


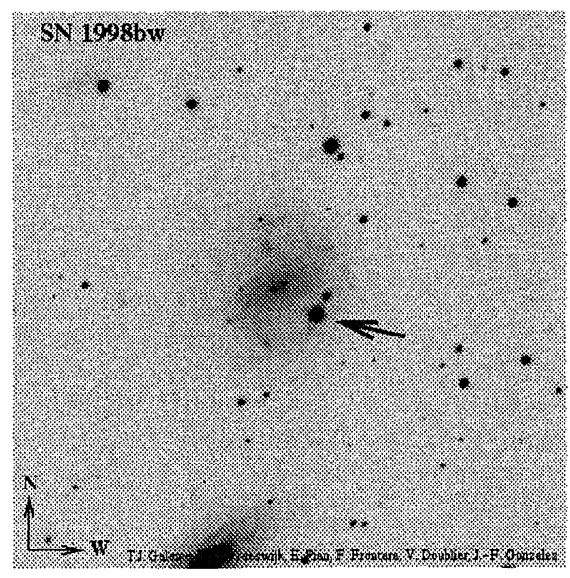

FIGURE 6. Discovery image of SN 1998bw (reprinted by permission from Nature [30] copyright (1998) Macmillan Magazines Ltd.). The $S N$ is the bright object (marked with an arrow) $S W$ of the nucleus. Relative to typical $S N e$, this $S N$ is more energetic and appears to have synthesized ten times more Nickel.

flux $\propto \rho^{1 / 2}$ ) that are potentially detectable. Radio observations have additional advantages of a longer lived afterglow, immunity from weather and freedom from the diurnal cycle.

Gamma-Ray Bursts Associated with Supernovae. Observers and theorists alike have been intrigued by the possibility that the bright supernova, SN 1998bw, discovered by Galama et al. [30] in the error circle of GRB 980425 [67], is associated with the gamma-ray event (Figure 6). Kulkarni et al. [47] discovered that the SN had an extremely bright radio counterpart; see Figure 7. We noted that the inferred brightness temperature exceeded the inverse Compton catastrophe limit of $5 \times$ $10^{11} \mathrm{~K}$ and to avoid rapid cooling we postulated the existence of a relativistically expanding blastwave $(\Gamma \gtrsim 2)$. This relativistic shock is, of course, in addition to the usual sub-relativistic SN shock. This relativistic shock may have produced the GRB at early times. (We note here that we disagree with the much lower energy estimates of [81]; our recent calculations using the same assumptions as those made in [81] result in an energy estimate similar to that obtained earlier [47] from minimum-energy formulation). The optical modeling of the lightcurve and the spectra show that GRB 980425 was especially energetic $[43,86]$ with an energy release of $3 \times 10^{52} \mathrm{erg}$ and Nickel production of nearly nearly a solar mass.

If GRB 980425 is associated with SN 1998bw, then this type of event is rare among the SAX localizations. GRB 980425 is most certainly not a typical GRB: the redshift of SN 1998bw is 0.0085 and the $\gamma$-ray energy release in GRB 980425 is at least four orders of magnitude less than in other cosmologically located GRBs. For this reason, most astronomers (especially those in the GRB field; see Wheeler's foray in experimental sociology [82]) do not believe the association between GRB 980425 and SN 1998bw. On the other hand, as evidenced by the intense interest in and modeling of the radio and optical data of SN 1998bw, this object is of considerable interest to the SN community. Indeed, we believe that the proposed GRB-SN association controversy has muddied the main issue: SN 1998bw is an interesting 


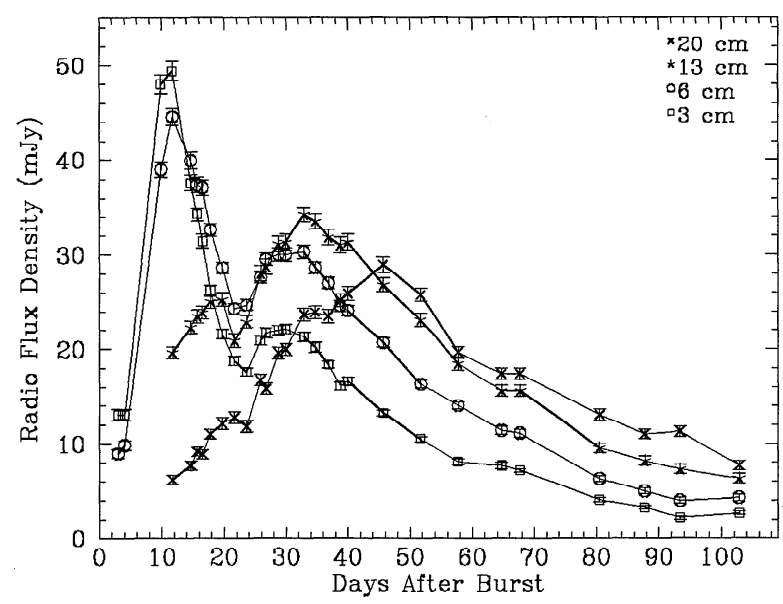

FIGURE 7. The radio light curve of $S N 1998 b w$ at four wavelengths (reprinted by permission from Nature [47] copyright (1998) Macmillan Magazines Ltd.). The peak brightness temperature of SN 1998bw at early times is $10^{13} \mathrm{~K}$, well in excess of the inverse Compton limit of $5 \times 10^{11} \mathrm{~K}$, and can be best understood if the radio emission originates from a relativistic shock $(\Gamma \gtrless 2)$.

SN in its own right.

What is the true distinguishing feature of SN 1998bw that may connect it to a GRB event? Is it the large energy release, as suggested by several authors $[43,33]$ ? We argue that in fact it is the energy coupled into relativistic ejecta that most closely connects SN $1998 \mathrm{bw}$ to a GRB. In a typical SN, about $10^{51}$ erg is coupled to the envelope of the star (a small fraction of the total SN energy release of $10^{53}$ erg). In a GRB, a similar amount of energy $\left(10^{51}-10^{52}\right.$ erg depending on the event) is coupled to a much smaller ejecta mass, resulting in relativistic outflow. For SN 1998bw, applying the minimum energy formulation to the radio observations we infer the relativistic shell to contain $\sim 10^{50} \mathrm{erg}$. Not only is this uncharacteristic of a typical SN (there exists no evidence for relativistic ejecta in ordinary SN), but it is not dissimilar from the energy implied for GRB outflows. One could therefore envisage a continuum of physical phenomenon between SN 1998bw and cosmological GRBs provided we use the energy in the relativistic ejecta as the basic underlying parameter and not the isotropic gamma-ray release.

\section{AFTERGLOW: THE PHYSICS AND ENERGETICS OF THE FIREBALL}

One can consider a GRB to be like a SN explosion with a central source releasing energy $E_{0}$ (comparable to the mechanical release of energy in an $\mathrm{SN}$ ). This is the socalled fireball model. The difference between an SN and a GRB is primarily in ejecta 
mass: $1-10 M_{\odot}$ for SNe whereas only $10^{-5} M_{\odot}$ for GRBs. The evolution of a GRB is much faster than that of a SN due to two factors: the ejecta expand relativistically and, thanks to the smaller ejecta mass, the optical depth is considerably smaller.

As the ejecta encounter ambient gas, two shocks are produced: a short-lived reverse shock (traveling through the ejecta) and a long-lived forward shock (propagating into the swept-up ambient gas). Afterglow emission is identified with emission from the forward shock. In order to obtain significant afterglow emission, several conditions are necessary. (1) Rapid equipartition of electrons with the shocked protons (which hold most of the energy). (2) Acceleration of electrons to a power-law spectrum (particle Lorentz factor distribution, $d N / d \gamma \propto \gamma^{-p}$ ). (3) Rapid growth of the magnetic field with energy density in the range of $10^{-2}$ of that of the protons. Under these circumstances, afterglow emission is dominated by synchrotron emission of the accelerated particles; see $[71,79]$. The weakness of this model is the assumption of growth in the magnetic field strength to the high values noted above (R. Blandford, pers. comm.).

The theoretically expected afterglow spectrum is shown in Figure 8. Three key frequencies can be identified: $\nu_{a}$, the synchrotron self-absorption frequency; $\nu_{m}$, the frequency of the electron with a minimum Lorentz factor (corresponding to the thermal energy behind the shock) and $\nu_{c}$, the cooling frequency. Electrons which radiate above $\nu_{c}$ cool on timescales equal to the age of the shock. The evolution of these three frequencies is determined by the hydrodynamical evolution of the shock, which in turn is affected by two principal factors: the environment of the GRB and the geometry of the explosion.

The GRB environment. The earliest afterglow models made the simplifying assumption of expansion into a constant density medium. This is an appropriate assumption should the GRB progenitor explode into a typical location of the host galaxy. However, there is increasing evidence tying GRBs to massive stars (see $\S I I I)$. It is well known that massive stars lose matter throughout their lifetime and thus one expects the circumburst medium to exhibit a density profile, $\rho \propto r^{-2}$ where $r$ is the distance from the progenitor. Chevalier \& Li [8] refer to these two models as the ISM (interstellar medium) and the wind model respectively. As can be seen from Figure 8 these two models give rise to rather different evolution of the three critical frequencies.

Geometry: Jets versus Spheres. The hydrodynamics is also affected by the geometry of the explosion. Many powerful astrophysical sources have jet-like structure. There is evidence (from polarization observations) indicating asymmetric expansion in $\mathrm{SNe}$ [82], so it is only reasonable to assume that GRB afterglows also have jet-like geometry as well. A clear determination of the geometry is essential in order to infer the true energy of the explosion. This is especially important for energetic bursts such as GRB 990123 whose isotropic energy release approaches $M_{\odot} c^{2}$.

Let the opening angle of the jet be $\theta_{0}$. As long as the bulk Lorentz factor, $\Gamma$, is larger than $\theta_{0}^{-1}$, the evolution of the jet is exactly the same as that of a sphere (for an observer situated on the jet axis). However, once $\Gamma$ falls below $\theta_{0}^{-1}$ then two 
effects become important. First, for a well defined jet, the on-axis observer sees an edge and thus one expects to see a break in the afterglow emission. Second, the lateral expansion of the jet (due to heated and shocked particles) will start affecting the hydrodynamical explosion.

Wind or ISM? The two key diagnostics to distinguish these two models are the evolution of the cooling frequency (see Figure 8) and the early behavior of the radio emission. In the wind model, the radio emission rises rapidly (relative to the ISM model) and the synchrotron self-absorption frequency falls rapidly with time. Both these result from the fact that the ambient density decreases with radius (and hence in time) in the wind model.

Unfortunately, in general, the current data are not of sufficient quality to firmly distinguish the two models. For example in GRB 980519, the same optical and X-ray data appear to be adequately explained by the jet+ISM model [73] and the sphere+wind model [8]. Including the radio data tips the balance, but only slightly, in favor of the wind model [23]. In our opinion, the best example for the wind model is that of GRB 980329 [20]; see Figure 9. This afterglow exhibits the two unique signatures of the wind model: high $\nu_{a}$ and a rapid rise. Given the importance of making the distinction between the wind and the ISM model, we urge early wide band radio observations (especially at high frequencies).

Energetics. Of all the physical parameters of the fireball, the most eagerly sought parameter is the total energy $E_{0}$. By analogy with supernovae, it is $E_{0}$ which sets the GRB phenomenon apart from other astrophysical phenomena. Classes of GRBs

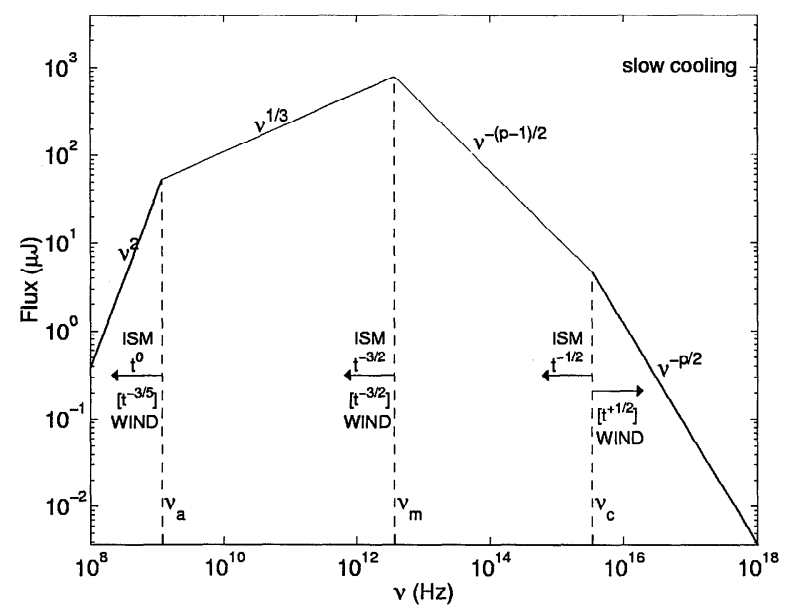

FIGURE 8. Broad-band spectrum $\left(f_{\nu}\right)$ of the afterglow from a spherical fireball with constant density ("ISM" model; see text) and $\rho \propto r^{-2}$ medium ("wind" model; see text). This is representative of the observed spectrum a few days after the burst. Note the distinct evolution of $\nu_{a}$ and $\nu_{c}$ in the two models. 

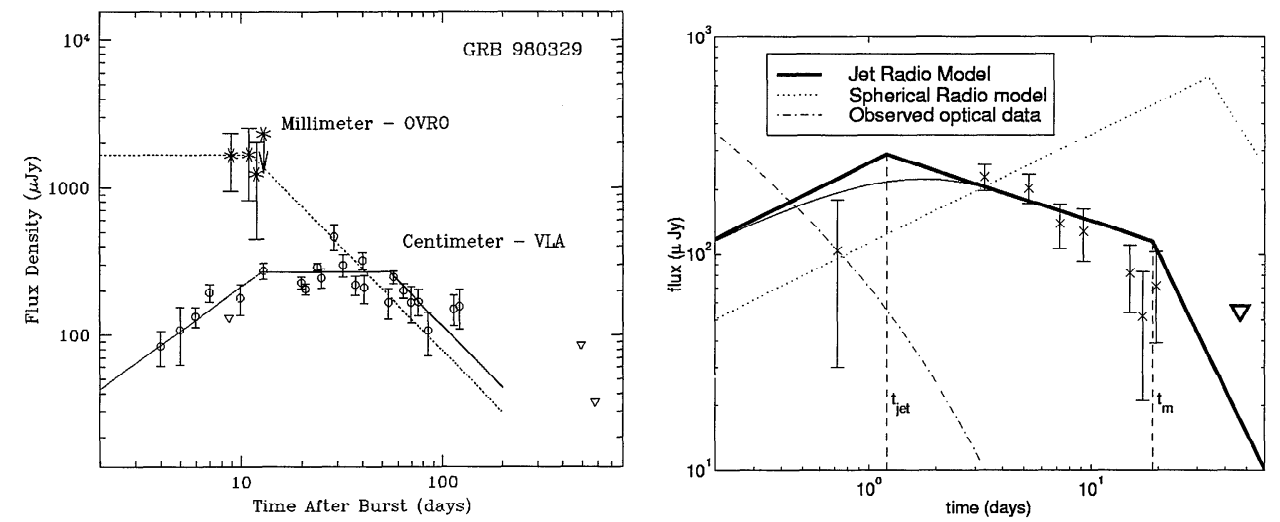

FIGURE 9. Left: Radio afterglow of GRB 980329 [20]. The rapid rise of the centimeter flux and the high absorption frequency (signified by the considerable strength of the millimeter emission) offer good support for GRB 980329 expanding into a circumburst medium with density falling as inverse square distance. The lines represents a wind model based on X-ray, optical, IR, $\mathrm{mm}$ and cm data. Right: Observed and model radio light curves of GRB 990510 [39]. The model predictions for the radio afterglow emission are displayed by the solid line (jet fireball model) and dotted line (spherical fireball model). The observed optical afterglow emission is displayed by the dotted-dashed line; see text for more details.

may eventually be distinguished and ranked by their energy budget; for example, long-duration events, short duration events and supernova-GRBs (see $\S I I I)$.

One approach has been to use the isotropic $\gamma$-ray energy as a measure of $E_{0}$; see Figure 3. There are three well known problems with such estimates. First, collimation of the ejecta (jets) will result in overestimation of the total energy release. For GRB 990510, where a good case for a jet has been established (Figure 9), the standard isotropic energy estimate is probably a factor of 300 more than the true energy [39]. Second, even after accounting for a possible jet geometry, the efficiency of converting the shock energy into gamma-ray emission is very uncertain. For example, some authors [51] advocate low efficiency $(\sim 1 \%)$, which would result in an enormous upward correction to the usual isotropic estimates. Third, the bulk Lorentz factor is extremely high during the emission of $\gamma$ rays and thus the estimates critically depend on assumption of the geometry and granularity [52] of the emitting region. In particular, if the emission is from small blobs [52] then the inferred estimates are grossly in error.

In contrast to this highly uncertain situation, afterglows offer (in principle) more robust methods to evaluate $E_{0}$. In view of the importance of determining $E_{0}$ we summarize the different methods of determining $E_{0}$ from afterglow observations. One approach is to fit a "snapshot" broad-band afterglow spectrum (from radio to $\mathrm{X}$ rays) to an afterglow model; this approach was pioneered by Wijers \& Galama [83]. The strength of this method is that the estimated $E_{0}$ is, in principle, robust. 
Specifically, the estimate does not depend on the usually unknown environmental factors (run of density). However, in practice, this method is very sensitive to the values of the critical frequencies (Figure 8) which are usually not well determined. This difficulty explains the wildly differing estimates of $E_{0}$ for GRB $970508[83,35]$. Furthermore, this method uses measurements obtained at early times (when the afterglow at high frequencies is bright) with the result that the true source geometry is hidden by relativistic beaming.

A second approach is to model the light curves of the afterglow in a given band, specifically a radio band. The advantages of this method are the photometric stability of radio interferometers and the low Lorentz factor at the epoch of the peak of the radio emission. The disadvantages are two-fold: the sensitivity to the environmental parameters (density) and the assumption of the constancy of the microphysics parameters (electron and magnetic field equipartition factors). Application of this approach to GRB 980703 has resulted in seemingly accurate measures of the fireball parameters [19].

Freedman \& Waxman [24] take yet another approach, and estimate the energy release from late time X-ray observations. They show that the X-ray flux is insensitive to the GRB environment, and obtain robust estimates of the fireball energy per unit solid angle: from $3 \times 10^{51} \mathrm{erg}$ to $3 \times 10^{53} \mathrm{erg}$.

With all the above approaches, however, the possible collimation of the ejecta in jets is still a major uncertainty. This can be addressed by observing the evolution of the afterglow as the "edge" of the jet becomes visible. In most cases no evidence for jets has been seen, with the notable exceptions of GRB 990510 and possibly GRB 990123. In addition, a variety of statistical arguments (the absence of copious numbers of "orphan afterglows") $[37,36,70]$ suggests that, on average, the collimation cannot be extreme, and that for most bursts the opening angle is not less than 0.1 radian. Thus, the total energy for most bursts may be reduced to the range of $10^{50} \mathrm{erg}$ to $3 \times 10^{51} \mathrm{erg}$, but could easily be much higher in at least some cases.

Possibly the best approach to determining the energetics, which minimizes uncertainties due both to collimation (jets) and to the environment, is to model the afterglow after it becomes non-relativistic. This method builds on the well-established minimum energy formulation and the self-similarity of the Sedov solution. Not only are the ejecta truly non-relativistic, but they are also essentially spherical, as by this time jets will have had sufficient time to have undergone significant lateral expansion. Indeed, we can justifiably call this "fireball calorimetry" [21]. Applying this technique to the long-lived afterglow of GRB 970508 (Figure 1) led to the surprising result that $E_{0} \sim 5 \times 10^{50} \mathrm{erg}$ - weaker than a standard SN! This is an astonishing result. If true, this result would suggest that it is not $E_{0}$ which is the prime distinction between GRBs and SNe but the ejecta mass. However, Chevalier \& $\mathrm{Li}[9]$ interpret the same data in the wind framework and derive much larger $E_{0}$. Clearly, we need more well-studied afterglows with sufficient observations to first distinguish the circumburst environment (wind versus ISM) and then radio observations over a sufficiently long baseline to undertake calorimetry. Nonetheless, one 
should bear in mind that the current evidence for large energy release in GRBs is not as strong as is usually assumed.

\section{EPILOGUE AND FUTURE}

Clearly, the GRB field is evolving rapidly. Along what direction[s] will this field proceed in the coming years? One way to anticipate the future is by considering analogies from the past.

In §III we already discussed the parallels between the SN field and the GRB field. Here we discuss the numerous parallels with quasar astronomy. First discovered at radio wavelengths, we now study quasars across the electromagnetic spectrum. Although still identified by their gamma-ray properties, we now recognize the tremendous value of pan-chromatic GRB and afterglow studies. In both cases, there was considerable controversy about the distance scale. However, once this issue was settled, it became clear that quasars are the most energetic objects (sustained power) whereas GRBs are the most brilliant. For both, the ultimate energy appears to be related to black holes (albeit of different masses).

The raging issues in GRB astronomy today are the same that fueled quasar studies in the 60's: the spatial distribution, the extraction of energy from the central engine, the transfer of energy from stellar scales to parsec scales, and the geometry of the relativistic outflow (sphere or jet). Astronomers took decades to unify the seemingly diverse types of quasars, and to conclude that there are two types of central engines: radio loud and radio quiet. Likewise, there may well be two types of GRB engines: rapidly and slowly spinning black holes emerging respectively from collapse of a rotating core of a massive star or coalescence of compact objects and the collapse of a massive star. This picture could potentially explain both the cosmologically located GRBs and SN 1998bw. Finally, we can project that in the future, GRBs may be used to probe distant galaxies, just as quasars are used today to study the IGM.

There is a feeling in the astronomical community (outside the GRB community) that the GRB problem is "solved". The truth is that the GRB problem is now getting defined! We now summarize our view of the major issues and anticipated near-term advances. In our opinion the major issues are Diversity, Progenitors and Energy Generation.

As discussed earlier, high energy observations suggest the existence of two classes: short and long-duration bursts. It is possible that afterglow observations may demarcate additional classes. If so, one can contemplate that within a year (assuming abundant localizations by HETE-2) that we will have new GRB designations such as $s$ GRBs (GRBs with late time bump indicative of an underlying SN), $w$ GRBs (GRBs whose afterglow clearly indicates a wind circumburst medium shaped by stellar winds), $i$ GRBs (GRBs which explode in the interstellar medium) and so on.

The broad indications are that GRBs are associated with stars and most likely massive stars. However, we know little beyond this. Comparing the unbeamed GRB 
event rate of $1.8 \times 10^{-10} \mathrm{yr}^{-1} \mathrm{Mpc}^{-3}$ [74] with $3 \times 10^{-5}$ Type Ibc SN yr ${ }^{-1} \mathrm{Mpc}^{-3}$ and $10^{-6} \mathrm{yr}^{-1} \mathrm{NS}-\mathrm{NS}$ merger $\mathrm{Mpc}^{-3}$ [53] shows that GRBs events are extremely rare; here we note that the present data do not support a collimation correction in excess of 100 . It will be quite some time before we will be in a position to identify the conditions necessary for a star to die as a GRB.

It is our opinion that SN 1998bw is a major development in the field of stellar collapse. The association (or lack) with GRB 980425 unfortunately has distracted our attention of this important development. The existence of a significant amount of mildly relativistic material, $\sim 10^{50} \mathrm{erg}$ [47], is fascinating and it is ironic that none of the models can account for this inferred value whereas most of the theoretical effort has gone into explaining the gamma-ray burst itself (especially considering the uncertain association of GRB 980425 with SN 1998bw). Clearly, SN 1998bw is a rare event, but we are convinced that more such events will be found and accordingly have mounted a major campaign to identify these SNe. The robust signatures of this class are high $T_{B}$ and prompt X-ray emission since these are necessary consequences of a relativistic ejecta. We note that if these future events are as bright as SN 1998bw then the energy in the relativistic ejecta can be directly measured by VLBI observations of the expanding radio shell.

It is vitally important to make quantitative progress in determining the energy release in GRBs. As discussed in §IV, firm estimates of the energy release require well-sampled broad-band data at early times and densely sampled radio light curves out to late times. This will require a coordinated approach and necessarily involve many observatories around the world and in space. The same datasets will also help us understand a profound puzzle: if GRBs indeed arise from the death of massive stars, then why do we not see signatures for a circumburst medium shaped by stellar winds in all long-duration GRBs? Even ardent supporters of the wind model $[8,9]$ concede that some GRBs (e.g., GRB 990123, GRB 990510) are due to a jet expanding into a constant density medium.

We now discuss the anticipated returns. True to our tradition as observers, we order the discussion by wavelength regimes!

Radio Observations: Dusty Galaxies, Circumstellar Edges and Reverse Shocks. Perhaps the most exciting use of radio afterglow is in identifying dusty star-forming host galaxies. Such host galaxies are not readily seen at optical wavelengths. Currently, such galaxies are eagerly sought and studied at sub-millimeter wavelengths. However, the sensitivity and localization of such galaxies by submillimeter telescopes is poor. In contrast, GRB host galaxies are identified at the sub-arcsecond level. The present radio afterglow detection rate of $40 \%$ already places an upper limit on the amount of star-formation in dusty regions, viz. this rate is not larger than that measured from optical observations. This result is entirely independent of the conclusion based on studies in the sub-millimeter regime, or the diffuse cosmic FIR background found in the COBE data. However, the result does rely on two assumptions: (i) GRBs trace star formation and (ii) the GRB explosion and its aftermath does not radically alter the ambient medium (i.e., with 
a prompt and complete destruction of dust grains along the line of sight).

Radio observations of SNe offer a probe of the distribution of the circumstellar matter. A spectacular example is SN $1980 \mathrm{~K}$ whose radio flux dropped $14 \mathrm{yr}$ after the explosion [58]. A progenitor star which suffered mass loss with variation in the wind speed could explain the observations. Indeed, one expects significant radial structure in the circumburst medium as the progenitor evolves from a blue star to a red supergiant and thence to possibly a blue supergiant, etc. If GRBs come from binary stars which undergo a phase of common envelope evolution [7] then the structure would be even more complicated. Thus, radio observations have the potential (in fortunate circumstances) to give us insight into the mass-loss history of the progenitor star(s).

The prompt optical emission from GRB 990123 [1] has been interpreted to arise from the reverse shock [72]. Far less discussed is the prompt radio emission - a radio flare - also seen from this burst [49]. Sari \& Piran [72] suggest that the radio emission also originates from the reverse shock as the electrons cool. Observations related to the reverse shocks are important since it is only through these observations that we have a chance of studying the elusive ejecta. We now have four such examples of radio flares [50] and this represents an order of magnitude better success rate than ROTSE+LOTIS. We urge theorists to pay attention to these new findings. More to the point, radio observations appear to be fruitful for the study of reverse shocks, especially when combined with observations of the prompt optical emission. This bodes well for the coming years given the efforts underway to increase the sensitivity of ROTSE [1].

X-ray Observations: Diversity \& Progenitors. GINGA identified a number of X-ray rich GRBs. BeppoSAX has found several such examples with some bursts lacking significant gamma-ray emission - the so-called X-ray flashes [40]. We know very little about these X-ray transients. Could they be GRBs in a very dense environment (with red giant progenitors)? We need to take such transients more seriously and intensively followup on such bursts.

Another interesting finding from GINGA was the discovery of precursor soft $\mathrm{X}$-ray emission [62]. There is no simple explanation for this phenomenon in the current internal-external shock model. We suggest that the soft X-ray emission precursor is similar to the UV breakout of ordinary SNe. This hypothesis can be confirmed or rejected by obtaining the redshift to such bursts.

The X-ray rich GRB $981226[26,18]$ was marked with two additional peculiarities: a precursor emission and afterglow emission which is seemingly undetectable after about 12 hours but then rises rapidly before commencing decay. Above we alluded to the fact that massive stars do not have a single phase of mass loss but instead have a veritable history of mass loss (from birth to death). The X-ray observations of GRB 981226 could be accounted for in a model in which the progenitor has first a red supergiant wind followed by a blue supergiant wind.

Optical Observations: SN Link, Short Bursts \& Geometry. The GRB-SN connection is best probed by optical observations. The value of optical observations 
has already been demonstrated by the current observations of GRB 980326 and GRB 970228. Clearly, more observations are needed to establish this link. Once this link is established then one can undertake detailed spectroscopic studies of the SN with large ground-based telescopes and photometric studies with HST.

Offsets of GRBs and the morphology of the host galaxies will continue to be of great interest. Such observations will help us differentiate whether some GRBs come from nuclear regions or always from star-forming regions. Under the current paradigm, the discovery of GRBs coincident with elliptical galaxies would be a major surprise. On the other hand, one expects short bursts to arise in the halo of their galaxies and thus, in this case, no coincidence is expected. We expect HETE-2 to contribute significantly to these issues. Finally, polarization measurements offer a very convenient way to probe the geometry of the emitting region as has already been demonstrated from the discovery of polarization in GRB 990510 (c.g., [55,84]).

\section{ACKNOWLEDGMENTS}

Our research is supported by NASA and NSF. JSB holds a Fannie \& John Hertz Foundation Fellowship, AD holds a Millikan Postdoctoral Fellowship in Experimental Physics, TJG holds a Fairchild Foundation Postdoctoral Fellowship in Observational Astronomy and RS holds Fairchild Foundation Senior Fcllowship in Theoretical Astrophysics. The VLA is a facility of the National Science Foundation operated under cooperative agreement by Associated Universities, Inc. The W. M. Keck Observatory is operated by the California Association for Research in Astronomy, a scientific partnership among California Institute of Technology, the University of California and the National Aeronautics and Space Administration. It was made possible by the generous financial support of the W. M. Keck Foundation.

\section{REFERENCES}

1. Akerlof, C., Balsano, R., Barthelmy, S., et al., Nature 398, 400 (1999).

2. Bloom, J. S., Djorgovski, S. G., Kulkarni, S. R. \& Frail, D. A., ApJ 508, L17 (1998).

3. Bloom, J. S., Sigurdsson, S. \& Pols, O. R., MNRAS 305, 763 (1999).

4. Bloom, J. S., et al., ApJ 518, L1, (1999).

5. Bloom, J. S., et al., Nature 401, 453 (1999).

6. Boella, G., et al., A.\&A. Suppl. Ser. 122, 298 (1997).

7. Brown, G. E., Lee, C. -H., Lee, H. K. \& Bethe, H. A., astro-ph/9911458 (1999).

8. Chevalier, R. A. \& Li, Z.-Y., ApJ 520, L29, (1999).

9. Chevalier, R. A. \& Li, Z. -Y., astro-ph/9908272 (1999).

10. Costa, E., et al., Nature 387, 783 (1997).

11. Djorgovski, S. G., Kulkarni, S. R., Bloom, J. S., Goodrich, R., Frail, D. A., Piro, L \& Palazzi, E., ApJ 508, L17 (1998).

12. Djorgovski, S. G. et. al., in preparation, (2000). 
13. Eichler, D., Livio, M., Piran, T., \& Schramm, D. N., Nature 340, 126 (1989).

14. Fishman, G. J. \& Meegan, C. A., Annu. Rev. Astron. Astrophys. 33, 415 (1995).

15. Frail, D. A. \& Kulkarni, S. R., Astrophys. Space Sci. 231, 277 (1995).

16. Frail, D. A., Kulkarni, S. R., Nicastro, S. R., Feroci, M., \& Taylor, G. B., Nature 389, 261 (1997).

17. Frail, D. A., Kulkarni, S. R., Shepherd, D. S., \& Waxman, E., ApJ 502, L119, (1998).

18. Frail, D. A., et al., ApJ 525, L81 (1999).

19. Frail, D. A., Bloom, J. S., Kulkarni, S. R., Sari, R., \& Taylor, G. B., in preparation, (2000).

20. Frail, D., Kulkarni, S., Sari, R., Taylor, G., Shepherd, D., Bloom, J., Young, C., Nicastro, L., \& Masetti, N., $A p J$ in press, (1999).

21. Frail, D., Waxman, E. \& Kulkarni, S. R., $A p J$ in press, (2000).

22. Frail, D. A., Kulkarni, S. R., Wieringa, M. H., et al., astro-ph/9912171, (1999).

23. Frail, D. A., Kulkarni, S. R., Sari, R., et al., astro-ph/9910060, (2000).

24. Freedman, D. L. \& Waxman, E., astro-ph/9912214 (1999).

25. Frontera, F., Amati, L., Costa, E., et al., astro-ph/9911228, (1999).

26. Frontera, F., Antonelli, L. A., Amati, L., et al., ApJ, submitted (2000).

27. Fruchter, A. S., ApJ 512, L1 (1999).

28. Fruchter, A. S., Thorsett, S., Metzger, M. R., ApJ 519, L13 (1999).

29. Fruchter, A. S., Pian, E., Thorsett, S. E., et al., $A p J$ 516, 683 (1999).

30. Galama, T. J., et al., Nature 395, 670, (1998).

31. Galama, T. J. et al., ApJ in press, (1999).

32. Garnavich, P. M. et al., ApJ 493, L53 (1998).

33. Germany, L., Reiss, D. J., Sadler, E. M., Schmidt, B. P. \& Stubbs, C. W., astro$\mathrm{ph} / 9906096$ (1999).

34. Gorosabel, J., Castro-Tirado, A. J., Pedrosa, A., et al., A.\&A. 347, L31 (1999).

35. Granot, J., Piran, T. \& Sari, R., ApJ 527, 236 (1999).

36. Greiner, J., et al., $A \& A S \mathbf{1 3 8}, 441$ (1999)

37. Grindlay, J. E., ApJ 510, 710 (1999)

38. Groot, P. J., Galama, T. J., van Paradijs, J., et al., ApJ 493, L27 (1998).

39. Harrison, F. A., et al., ApJ 523, L121 (1999).

40. Heise, J., talk at the 5th Hunstville GRB conference, (1999).

41. Holland, S. \& Hjorth, J., $A \& A$ 344, L67 (1999).

42. In't Zand, J. J. M., Amati, L., Antonelli, L. A., et al., ApJ 505, 1191 (1998).

43. Iwamoto, K., et al., Nature 395, 672, (1998).

44. Katz, J. I., ApJ 422, 248 (1993).

45. Katz, J. I. \& Canel, L. M., ApJ 471, 915 (1996).

46. Kulkarni, S. R., Djorgovski, S. G., Ramaprakash, A. N., et al., Nature 393, 35 (1998).

47. Kulkarni, S. R., Frail, D. A., Wieringa, M. H., et al., Nature 395, 663 (1998).

48. Kulkarni, S. R., Djorgovski, S. G., Odewahn, S. C., et al., Nature 398, 389 (1999).

49. Kulkarni, S. R., et al., ApJ 522, L97 (1999).

50. Kulkarni, S. R. \& Frail, D. A., in preparation, (2000).

51. Kumar, P., astro-ph/9907096 (1999). 
52. Kumar, P. \& Piran, T., astro-ph/9909014 (1999).

53. Lamb, D. Q., astro-ph/9909026 (1999).

54. Lattimer, J. M. \& Schramm, D. N., ApJ 192, L145 (1974).

55. Lazzati, D., Covino, S. \& Ghisellini, G., astro-ph/9912247 (1999).

56. Li, L.-X. \& Paczynski, B., ApJ 507, L59, (1998).

57. Macfadyen, A. I. \& Woosley, S. E., ApJ 524, 262, (1999).

58. Montes, M. J., van Dyk, S. D., Weiler, K. W., Sramek, R. A. \& Panagia, N., ApJ 506, 874, (1998).

59. Mészáros, P. \& Rees, M. J., ApJ 476, 232 (1997).

60. Metzger, M. R., Djorgovski, S. G., Kulkarni, S. R., Steidel, C. C., Adelberger, K. L., Frail, D. A., Costa, E., \& Frontera, F., Nature 387, 879 (1997).

61. Mochkovitch, R., Hernanz, M., Isern, J., \& Martin, X., Nature 361, 236-238, (1993).

62. Murakami, T., et al., Nature 350, 592 (1991).

63. Narayan, R., Paczyński, B., \& Piran, T., ApJ 395, L83 (1992).

64. Palazzi, E., et al., A.छ̈A. 336, L95 (1998).

65. Paczyński, B. \& Rhoads, J., ApJ 418, L5 (1993).

66. Pian, E., Fruchter, A. S., Bergeron, L. E., et al., ApJ 492, L103 (1999).

67. Pian, E. et al., A\&AS 138, 463 (1999).

68. Reichart, D. E., ApJ 521, L111 (1999).

69. Reichart, D. E., Lamb, D. Q., Metzger, M. R., et al., $A p J$ 517, 692 (1999).

70. Rhoads, J. E., ApJ 487, L1 (1997)

71. Sari, R., Piran, T. \& Narayan, R., ApJ 497, L17 (1998).

72. Sari, R. \& Piran, T., ApJ 517, L109 (1999).

73. Sari, R., Piran, T., \& Halpern, J. P., ApJ 519, L17 (1999).

74. Schmidt, M., ApJ 523, L117 (1999).

75. Strohmeyer, T. E., Fenimore, E.E., Murakami, T. \& Yoshida, A., Ap.J 500, 873 (1998).

76. Taylor, G. B., Frail, D. A., Kulkarni, S. R., et al., $A p J$ 502, L115 (1998).

77. van Paradijs, J., Groot, P. J., Galama, T., et al., Nature 368, 686 (1997).

78. Vietri, M., ApJ 488, L105 (1997).

79. Waxman, E., $A p J 489$, L33 (1997).

80. Waxman, E., \& Draine, B. T., astro-ph/9909020 (1999).

81. Waxman, E. \& Loeb, A., ApJ 515, 721 (1999).

82. Wheeler, J. C., astro-ph/9912403, (1999).

83. Wijers, R. A. M. J. \& Galama, T. J., ApJ 523, 177, (1999)

84. Wijers, R. A. M. J., Vreeswijk, P. M., Galama, T. J., et al., astro-ph/9906346 (1999).

85. Woosley, S. E., ApJ 405, 273, (1993).

86. Woosley, S. E., Eastman, R. G. \& Schmidt, B. P., ApJ 516, 788 (1999). 